\title{
Modelling and Analysis of CdS/CZTSSe Based Thin Film Solar Cell
}

\author{
M. Mehedi Hasan ${ }^{1}$, M. Iqbal Bahar Chowdhury ${ }^{2}$ \\ 1. Institute of Energy, University of Dhaka, Dhaka, Bangladesh \\ 2. Department of EEE, United International University, Dhaka, Bangladesh \\ Email:021mehedi.hasan@gmail.com ${ }^{1}$, ibcy@eee.uiu.ac.bd ${ }^{2}$
}

Received: 28 June 2018; Accepted: 9 September 2018; Available online: 25 December 2018

\begin{abstract}
Solar cells with a High efficiency and desire characteristics are modelled by considering the characteristics of the materials. An earth-abundant material Cu2ZnSn2(S, Se)4 (CZTSSe) could be a wise choice for the solar cell material with a band gap of $1.24 \mathrm{ev}$. In this paper, we made a model and try to represent an analysis of effect on $\mathrm{J}-\mathrm{V}$ characteristics and quantum efficiency of CdS/CZTSSe based solar cell during varying material and optical properties includes emitter width, carrier life time and resistances. From this model we can easily understand the effect of material and optical properties and graphical analysis of proposed solar cell.
\end{abstract}

Keywords: J-V Characteristics; Quantum efficiency; Carrier life time; material and Optical properties.

\section{Introduction}

The advance of solar cell as a solar panel has been rising day by day. In household use, charging electric vehicles, in space shuttle and as a micro $(<100 \mathrm{Kw})$ or $\operatorname{mini}(<10 \mathrm{Mw})$ grid is now a better alternative. That's why when we think about the alternative of grid line we can imagine that the answer is almost the solar cell. So, the performance of a solar cell must be at its peak. A solar cell with a desirable efficiency depends on its design parameter. In the case of designing a solar cell for the commercial purpose the cost for cell decided by the buyers. So, designing a solar cell with a higher efficiency is really call for choosing the right materials and right parameters. Our work describes analytical model of effect on efficiency when we vary the parameters. This paper also shows a tabular result of variations. Design of a solar cell depend many important factors which determine the efficiency, performance parameters as well as the cost (manufacturing). Materials of solar cell is one of the most important factor because depending on material we can vary the efficiency, cost, lifetime and the size of the panel. And our model gives us the right to choose a material as we want. Now how we will understand that what type of material will be used for as we want. For an example practice we choose CdS/CZTSSe structure [1]. The earth abundant and a low cost $\mathrm{Cu} 2 \mathrm{ZnSn}$ (Se, S)4 alloy is more efficient material for absorber layer for thin film solar cells [1,2,3]. Its high absorption coefficient makes it more able to absorb sun light in less thickness [2]. Gives a high efficiency compare to the lower thickness recorded as $12.6 \%$ [4]. CZTSSe shows a value of absorption coefficient up to 1400 $\mathrm{nm}$ which allow to absorb almost the full range of solar radiation [5]. As a result, we can have a chance to reduce the manufacturing cost that will be our goal. CZTSSe shows a combinational result of CZTS and CZTSe. Where CZTS has band gap of 1.5 and for CZTSe is 1[6]. And these two materials also have all the quality to be count as a concerning material for solar cell [7]. For the electron affinity CZTS and CZTSe shows 4.5 and 4.35 respectively [2]. As TCO ZnO make possible absorption of UV lights while having a band gap of 3.37ev [8]. Another advantage of $\mathrm{ZnO}$ is absorption of infrared light [9]. For more accurate value the $\mathrm{n}$ and $\mathrm{k}$ for $\mathrm{ZnO}$ can be found in [10]. In the emitter layer CdS give us the advantage of high band gap which allow a wide range of light absorption. It has a large-bandgap with a direct band gap of about $2.24 \mathrm{eV}$. As a result, CdS has the ability to absorb UV radiation due to band-to-band transitions. All this study shoes result of optical and electrical properties of the materials which is used to devices. Our work proposed a simple and interesting study on the effect of optical and the electrical parameters on the overall efficiency of a solar cell if we vary them. This study is based on the sensitive analysis of some controlling parameters of a Solar cell which affect directly on the efficiency.

\section{Proposed model}

Considering A full depleted structure in Fig.1, we can have a generalized equation for the total photo generated current $\left(J_{p h}\right)$ as stated in [11] is like

$$
J_{p h}=J_{p}\left(W_{e}\right)+J_{n}(0)
$$


where, $W_{e}$ is the emitter width, is the hole photo generated current density in the emitter region.

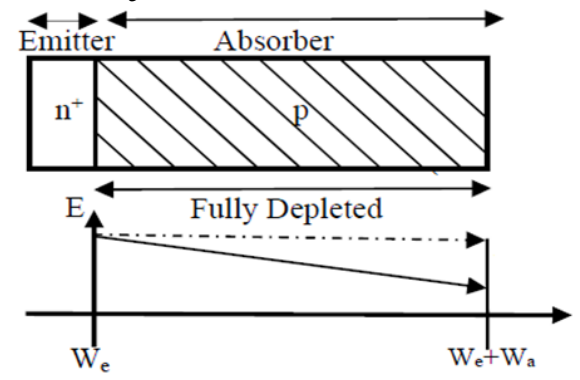

Figure 1: Electric field (E) [11]

The basic equation needs to deduce the electron current density $J_{n}(x)$ due to photo generation process can be given by,

$$
\begin{aligned}
& J_{n}(x)=q n(x) \mu_{n} E+q D_{n} \frac{d_{n}}{d_{x}} \\
& \frac{d J_{n}(x)}{d x}=\frac{q n(x)}{\tau_{n}}-q \alpha(\lambda) G_{0}(\lambda) e^{-\alpha(\lambda) x}
\end{aligned}
$$

As like,

$$
E=\frac{\varepsilon_{1} N_{d}}{\varepsilon_{1} N_{d}+\varepsilon_{2} N_{d}}\left[\frac{V_{b i}-\left(V_{L}-J_{L} R_{S}\right)}{W_{d}}\right]
$$

And

$$
G_{0}(\lambda)=(1-R) \frac{\lambda \mathrm{I}_{0}(\lambda)}{h c}
$$

A second order differential equation of $\mathrm{n}(\mathrm{x})$ can be obtained by combining equation (2) and (3), like

$$
\frac{d^{2} n}{d x^{2}}+c \frac{d n}{d x}-\frac{n}{L_{n}^{2}}=G e^{-\alpha_{2} x}
$$

The solution of the DE in (4) is,

$$
\mathrm{n}(\mathrm{x})=C_{1} e^{-\left(\frac{c}{2}-m_{0}\right) x}+C_{2} e^{-\left(\frac{c}{2}+m_{0}\right) x}-\mathrm{A} e^{-\alpha_{2} x}
$$

here the detail explanation of $\mathrm{C}, \mathrm{G}, \mathrm{G}_{0}, L_{n}$. From the Fig. 1 we get two boundary conditions as bellow.

$$
\frac{d n}{d x}(0) \approx 0
$$

And the other condition is to be,

$$
J_{n}\left(w_{a}\right)=q S_{n}\left(w_{a}\right)
$$

After evaluating the both boundary conditions we can calculate, $J_{n}(0)$ as,

$$
J_{n}(0)=q D_{n} n(0) \frac{E_{n}}{V_{T}}
$$

where, $\mathrm{n}(0)=C_{1}+C_{2}$. That gets the $J_{P h}$

\section{Results}

This section presents the simulation result carried out using the proposed model. First, the result of the proposed model has been verified against as experimental solar cell. This cell is a CZTSSe based thin film solar cell fabricated by Balakrishna Ananthoju et. el in work [1]. The layer thickness and the electrical parameters [1] for this structure are given in Table.1. In this work this structure has been simulated with the help of our proposed model. In doing so the following considerations made. First, the value of absorption coefficient $(\alpha)$ from absorbance (A). The second consideration is for the value of mobility of carriers. In the third position we assumed the absorber layer is fully depleted. Finally, since there was no doping information in the original paper, this parameter has been calculated using standard doping concentration for CZTSSe based structure. 
$\underline{\text { Table 1:Thickness of layer and electrical parameter [1] }}$

\begin{tabular}{ll}
\hline Parameter & Value \\
\hline TCO layer: $\mathrm{ZnO}$ & $300 \mathrm{~nm}$ \\
Buffer layer: $\mathrm{i}-\mathrm{ZnO}$ & $110 \mathrm{~nm}$ \\
Emitter layer: CdS & $60 \mathrm{~nm}$ \\
Absorber layer: CZTSSe & $1000 \mathrm{~nm}$ \\
Series resistance $\left(\mathrm{R}_{\mathrm{s}}\right)$ & $0.35\left(\mathrm{Ohm}-\mathrm{cm}^{2}\right)$ \\
Shunt resistance $\left(\mathrm{R}_{\mathrm{sh}}\right)$ & $\left(\mathrm{Ohm}-\mathrm{cm}^{2}\right)$ \\
\hline
\end{tabular}

Using these considerations, we figure out the simulation results using the proposed model have been compare with the experimental data of quantum efficiency and J-V characteristics. Fig.2. show the quantum efficiency and $\mathrm{J}-\mathrm{v}$ curve from simulation along with the experimental curve from [1]. From this comparison observation has been made that in the high wave length region of simulation data matches with experimental data, but in the low wave length region there has a difference. The main reason for this difference is that all data were not available so had to assume or approximate some data which is not directly equal to the experimental data. And considerations for the model may be was not like the experimental solar cell perfectly. From the Table. 2 comparison data with simulation data to the experimental data can be found.

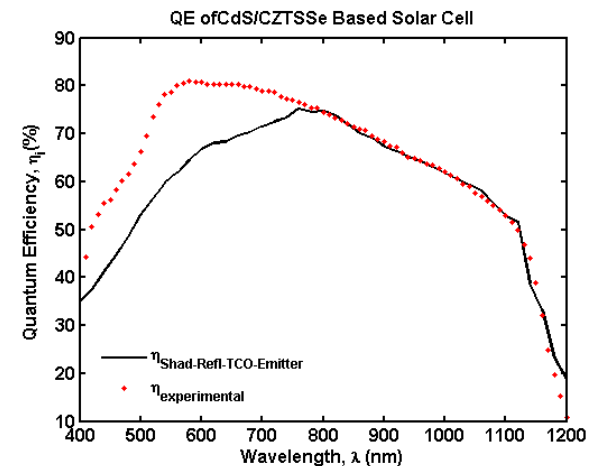

Figure 2(a): EQE $8.12 \%$ With FF 60\%

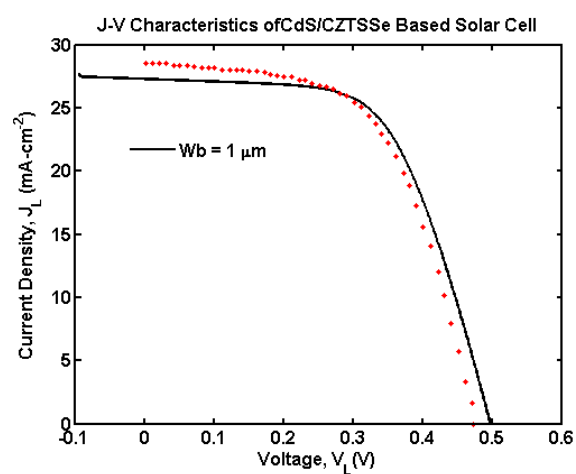

Figure 2(b): J-v curve with $\mathrm{V}_{\mathrm{oc}}$ of $0.4970 \mathrm{~V}$ and $\mathrm{J}_{\mathrm{sc}}$ of $27.47 \mathrm{~mA}$

Table 2: Compare with experimental data to Simulated data

\begin{tabular}{lll}
\hline Parameter & Experimental & Simulated \\
\hline ETA & $7.4 \pm .125 \%$ & $8.12 \%$ \\
Fill Factor & $59 \%$ & $60 \%$ \\
$\mathrm{~V}_{\text {oc }}$ & $476 \mathrm{mV}$ & $4970 \mathrm{mV}$ \\
$\mathrm{I}_{\mathrm{sc}}$ & $29 \mathrm{~mA} / \mathrm{cm}^{2}$ & $27.47 \mathrm{~mA} / \mathrm{cm}^{2}$ \\
\hline
\end{tabular}

Fig.3. show the variation of parameters based on change in emitter width. If Emitter width increases the number of recombination will increase as a result the carrier loss will increase which will occur decrease in short circuit current and energy loss. If energy decrease for recombination of carriers it will results the decrease in open circuit voltage because open circuit voltage is directly proportional to the energy. In Fig.3(b), show that open circuit voltage and short circuit current decrease with the emitter width increases. Table. 3 show the numerical change of parameters as a result of emitter width variation.

Table 4 depicted the change in $\mathrm{J}-\mathrm{V}$ characteristics for the electron life time variation.

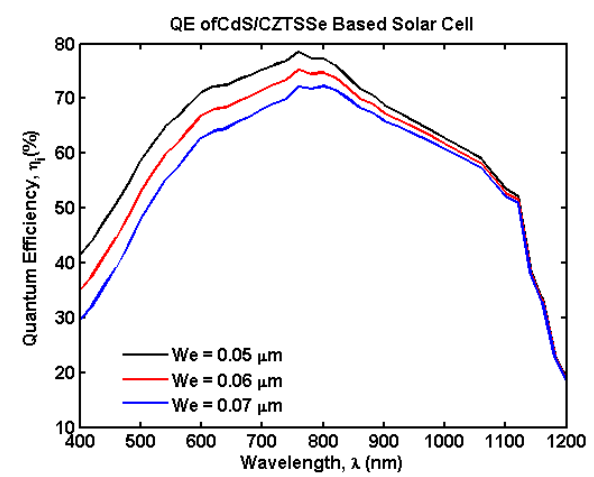

Figure 3(a): QE for Emitter width variation Figure

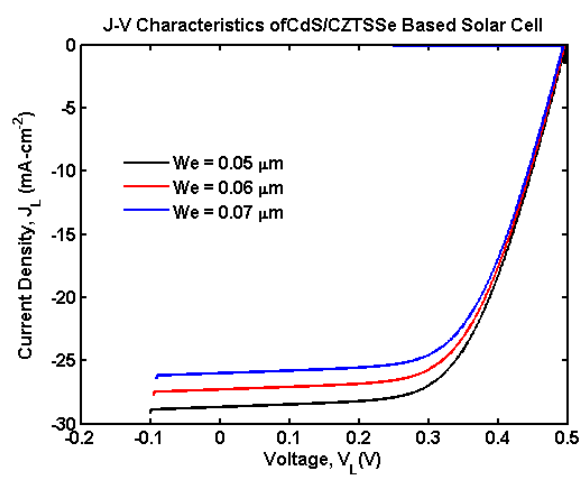

3(b): J-V characteristics for emitter width variation 
Table 3: Result for emitter width variation

\begin{tabular}{llll}
\hline Parameter & $\mathbf{W e}=. \mathbf{0 5} \boldsymbol{\mu m}$ & $\mathbf{W e}=.06 \boldsymbol{\mu m}$ & $\mathbf{W e}=.07 \boldsymbol{\mu m}$ \\
\hline $\mathbf{J}_{\mathrm{sc}}$ & 28.6748 & 27.2880 & 25.9996 \\
$\mathrm{~V}_{\mathrm{oc}}$ & 0.4980 & 0.4970 & 0.4950 \\
FF & 59.3893 & 59.8985 & 60.4835 \\
ETA & 8.4808 & 8.1235 & 7.7841 \\
\hline
\end{tabular}

From Fig.1 one thing which will be in consideration is that the distance which an electron has to cross in the depletion region is smaller, so by increasing the life time of electron it is possible that the will flow of electron through the circuit will increase. An important impact can be found from the property of Electron life time that recombination is inversely related with the electron life time that means increase in time duration of the electron will be able to increase the efficiency by reducing the percentage of recombination. By this means it will increase the short circuit current because of increase in carrier number and the open circuit voltage because of more energy.

Table 4: Result for electron life time variation

\begin{tabular}{clll}
\hline Parameter & $\boldsymbol{\tau n}=.0001 u s$ & $\boldsymbol{\tau n}=.001 u s$ & $\boldsymbol{\tau n}=\mathbf{1 u s}$ \\
$\mathrm{J}_{\mathrm{sc}}$ & 27.2524 & 27.2843 & 27.2880 \\
$\mathrm{~V}_{\mathrm{oc}}$ & 0.4960 & 0.4960 & 0.4970 \\
$\mathrm{FF}$ & 59.9990 & 60.0170 & 59.8985 \\
ETA & 8.1102 & 8.1221 & 8.1235 \\
\hline
\end{tabular}

As like the electron life time hole life is also inversely proportional to the recombination which can be shown in Fig.4. So, the overall efficiency will be increase as a result of increase in hole life time. But the distance has to cross by a hole in the depletion region is greater than the distance that crossed by an electron so variation in hole life time is more affective then variation in electron life time. This can be easily observed from the Fig.1. that distance travel by the hole is significantly very large then the distance travel by the electron that makes the difference between effect of variation of electron and hole though they are both carriers.

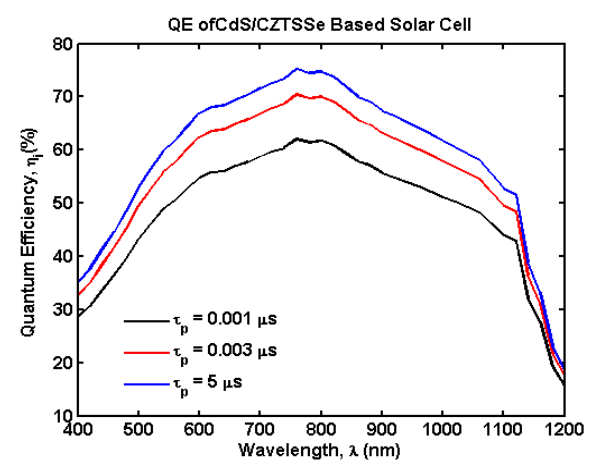

Figure 4(a): QE for Hole life time variation

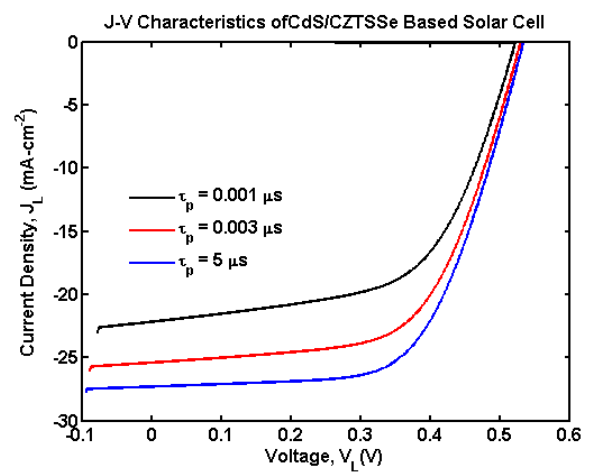

Figure 4(b): J-V characteristics for Hole life time variation

Table 5: Result for Hole life time variation

\begin{tabular}{clll}
\hline Parameter & $\boldsymbol{\tau p}=. \mathbf{0 0 1} \boldsymbol{\mu S}$ & $\boldsymbol{\tau p}=. \mathbf{0 0 3} \boldsymbol{\mu S}$ & $\boldsymbol{\tau p}=\mathbf{5} \boldsymbol{\mu S}$ \\
\hline $\mathrm{J}_{\mathrm{sc}}$ & 22.1638 & 25.3973 & 27.2987 \\
$\mathrm{~V}_{\mathrm{oc}}$ & 0.5220 & 0.5290 & 0.5330 \\
FF & 58.2468 & 60.5924 & 61.7789 \\
ETA & 6.7389 & 8.1407 & 8.9889 \\
\hline
\end{tabular}

Efficiency and the fill factor which is considered as the most important characteristics for output analysis depends on lifetime of charge carrier, mobility, diffusion length as those are the fundamental physical properties of solar cell materials. Here we consider Ideality factor as the most important indicator which is directly related to the output characteristics of solar cell. Another important parameter which is inversely proportional to the efficiency is recombination depends on the ideality factor. In the depletion region recombination depend on the ideality factor inversely. That means if ideality factor increases the rate of the recombination will decrease which is good for the total efficiency. Because if the rate of recombination decreases the carrier loss will decrease automatically. As a result, higher number of electron or hole will get the chance to increase the total energy which will increase the open circuit voltage as well as the short circuit current. 
Table 6: Result for Ideality Factor variation

\begin{tabular}{clll}
\hline Parameter & $\mathrm{IF}=1$ & $\mathrm{IF}=1.25$ & $\mathrm{IF}=1.5$ \\
\hline $\mathrm{J}_{\mathrm{sc}}$ & 27.2878 & 27.2880 & 27.2880 \\
$\mathrm{~V}_{\mathrm{oc}}$ & 0.3880 & 0.4970 & 0.5960 \\
$\mathrm{FF}$ & 57.9616 & 59.8985 & 62.0540 \\
ETA & 6.1368 & 8.1235 & 10.0922 \\
\hline
\end{tabular}

In Solar cells there are major two types of resistance that can affect the efficiency as well as the J-V curve. One is series resistance and the other is shunt resistance. Between this two-resistance shunt resistance can affect the efficiency more than the series resistance. Because a poor value of shunt resistance will give an alternative path to current flow that is not desirable. In Table.7 we can see the effect of $R_{s}$ on $J_{s c}$ and $V_{o c}$. An amount of dark current produce for series resistance which in term reduce the total current of solar cell. After all this increased reduction series resistance doesn't have lot significant on the total efficiency.

By analysing the effect of the parameters, a statement has been noted which will help one to understand the behaviour of a solar cell when we vary the emitter width, electron life time, hole life time, optical intensity, ideality factor, series resistance and shunt resistance. In our analysis, we also show the variation of J-V characteristics and on quantum efficiency. And we found that if we reduce the emitter width it increases the efficiency. Electron life time and hole life time in together carrier life time is directly proportional to the efficiency. But variation in hole life time is more effective than the variation in electron life time. we also observed that we can increase the efficiency by increasing the optical intensity as optical intensity is directly related to the carrier generation. More intensity means more carrier generation. Efficiency also can be increase by increasing the ideality factor. Series resistance has a little effect on solar cell but we cannot ignore the impact. Variation in shunt resistance vary the efficiency in a large scale. Now all our data from the analysis is in the table, from where we can easily find out the variation for each and every parameter individually.

Table 7: Result for Series (Rs) and Shunt (Rsh) Resistance variation

\begin{tabular}{lllllll}
\hline Parameter & $\mathrm{Rs}=.1$ & $\mathrm{Rs}=3.5$ & $\mathrm{Rs}=4.5$ & $\mathrm{R}_{\mathrm{sh}}=0.1 \mathrm{k}$ & $\mathrm{R}_{\mathrm{sh}}=0.2$ & $\mathrm{R}_{\mathrm{sh}}=0.5$ \\
\hline $\mathrm{J}_{\mathrm{sc}}$ & 27.4746 & 27.2880 & 27.2334 & 26.5491 & 27.0062 & 27.2880 \\
$\mathrm{~V}_{\mathrm{oc}}$ & 0.4980 & 0.4970 & 0.4720 & 0.4910 & 0.4950 & 0.4970 \\
$\mathrm{FF}$ & 74.1252 & 59.8985 & 58.8700 & 55.4853 & 58.2827 & 59.8985 \\
ETA & 10.1421 & 8.1235 & 7.5672 & 7.2329 & 7.7913 & 8.1235 \\
\hline
\end{tabular}

\section{Conclusion}

An example practice model has been used for the verification of our model. The analysis is used to observe effect of variation of different parameter which effect the efficiency directly. It is observed that for a given specification the value of parameters varies. A simulation result has been described to Choose the value of parameters for obtaining optimum efficiency. Finally, a new analysis of choosing parameters for variation in efficiency of solar cell has been developed. This analysis is simple and convenient because the measures to control the efficiency can be made by only selecting the right value of parameters in the model. The technique of varying the optical and material parameter has also been observed. Once the parameter get change, we should be able to calculate the new efficiency

\section{References}

[1] Balakrishna Ananthoju, Jeotikanta Mohapatra, Manoj K. Jangid, D. Bahadur, N. V. Medhekar \& M. Aslam, Cation /Anion Substitution in Cu2ZnSnS4 for Improved Photovoltaic Performance. Scientific Reports, 6:35369, DOI: $10.1038 /$ srep35369

[2] O.K. Simya, A. Mahaboobbatcha, K. Balachander, A comparative study on the performance of Kesterite based thin film solar cells using SCAPS simulation program, Superlattices and Microstructures 82 (2015) 248-261

[3] O.K. Simya, A. Mahaboobbatcha, K. Balachander, Compositional grading of CZTSSe alloy using exponential and uniform grading laws in SCAPS-ID simulation, Superlattices and Microstructures 92 (2016) 285-293

[4] Wei Wang, Mark T. Winkler, Oki Gunawan, Tayfun Gokmen, Teodor K. Todorov, Yu Zhu, and David B. Mitzi, Device Characteristics of CZTSSe Thin-Film Solar Cells with 12.6\% Efficiency, Advance Energy Material (2013) 1-5, DOI: 10.1002/aenm.201301465

[5] Wei Wu, Yanyan Cao, Jonathan V. Caspar, Qijie Guo, Lynda K. Johnson, Irina Malajovich, H. David Rosenfeld, Kaushik Roy Choudhury and Lee Silverman, Characterization of Printed CZTSSe Films for Photovoltaic Applications, Thin film solar technology V, SPIE, vol. 8823 88230G-1. 
[6] Clas Persson, Electronic and optical properties of Cu2ZnSnS4 and Cu2ZnSnSe4, Journal of Applied Physics 107, 053710 (2010), pp: 1-9.

[7] Shiyou Chen, X. G. Gong, Aron Walsh, and Su-Huai Wei, Crystal and electronic band structure of $\mathrm{Cu} 2 \mathrm{ZnSnX} 4$ (X = S and Se) photovoltaic absorbers: First-principles insights, Applied Physics Letters 94, 041903 (2009) pp: 1-4, DOI: 10.1063/1.3074499

[8] Ashour, M.A. Kaid, N.Z. El-Sayed, A.A. Ibrahim, Physical properties of ZnO thin films deposited by spray pyrolysis technique, ELSEVIER, Applied surface science 252 (2006), 7844-7848.

[9] D.G. Thomas, Infrared Absorption in Zinc Oxide Crystals, J. Phys. Chem. Solids Pergamon Press 1959. Vol. 10. pp. 47-51.

[10] N. Baydogan, T. Ozdurmusoglu, H. Cimenoglu, A.B. Tugrul, Refractive Index and Extinction Coefficient of ZnO: Al Thin Films Derived by Sol-Gel Dip Coating Technique, Defect and Diffusion Forum Vols. 334-335 (2013) pp 290-293

[11] Sanimul Alam Rivon, Protik Biswas, Abdullah Sakib Hasan, Ashiq Been Rahim, Md. Iqbal Bahar Chowdhury, Analytical Modelling of Cds/CdTe Based Thin Film Solar Cells Considering Surface Recombination, iCEEiCT (2016).

(C) 2018 by the author(s). This work is licensed under a Creative Commons Attribution 4.0 International License (http://creativecommons.org/licenses/by/4.0/). Authors retain copyright of their work, with first publication rights granted to Tech Reviews Ltd. 